TAIWANESE JOURNAL OF MATHEMATICS

Vol. 1, No. 2, pp. 143-148, June 1997

\title{
ON A CRITERION FOR MULTIVALENTLY STARLIKENESS
}

\author{
Yang Dinggong
}

\begin{abstract}
In this paper, we point out an error in [3, Main theorem] and obtain some sufficient conditions for multivalently starlikeness.
\end{abstract}

\section{INTRODUCTION}

Let $p \in N=\{1,2,3, \cdots\}$ and $A(p)$ denote the class of functions of the form

$$
f(z)=z^{p}+\sum_{n=p+1}^{\infty} a_{n} z^{n}
$$

which are analytic in the unit disk $E=\{z:|z|<1\}$.

A function $f(z)$ in $A(p)$ is called p-valently starlike if and only if

$$
\operatorname{Re} \frac{z f^{\prime}(z)}{f(z)}>0 \quad(z \in E) .
$$

We denote by $S(p)$ the subclass of $A(p)$ consisting of functions which are p-valently starlike in $E$.

There are many papers in which various sufficient conditions for multivalently starlikeness were obtained. Recently, Nunokawa [3, Main theorem] gave the following:

Theorem A. Let $f(z) \in A(p)$ and suppose that

$$
1+\frac{z f^{\prime \prime}(z)}{f^{\prime}(z)} \neq i b \quad(z \in E)
$$

where $b$ is a real number and

Received February 20, 1995

Communicated by M.-H. Shih.

1991 Mathematics Subject Classification: 30C45.

Key words and phrases: multivalently starlikeness, subordination. 


$$
|b| \geq 3^{1 / 2} p
$$

Then $f(z) \in S(p)$.

For $p=1$, the above result was also proved by Mocanu [2]. However, we find that Theorem $\mathrm{A}$ is not true when $p \geq 2$.

Counterexample. Let $p \geq 2$ and $f_{0}(z)$ be defined by

$$
f_{0}(z)=p \int_{0}^{z} \frac{t^{p-1}(1+t)^{p}}{(1-t)^{3 p}} d t \in A(p) .
$$

Then

$$
1+\frac{z f_{0}^{\prime \prime}(z)}{f_{0}^{\prime}(z)}=p\left(\frac{1+z}{1-z}+\frac{2 z}{1-z^{2}}\right) .
$$

Note that the univalent function

$$
w=\frac{1+z}{1-z}+\alpha \frac{2 z}{1-z^{2}} \quad(\alpha>0)
$$

maps $E$ onto the complex plane minus the half-lines $\operatorname{Re} w=0, \operatorname{Im} w \geq$ $(\alpha(\alpha+2))^{1 / 2}$ and $\operatorname{Re} w=0, \operatorname{Im} w \leq-(\alpha(\alpha+2))^{1 / 2}$ (see [2, p. 233]). From $(2)$, we have

$$
1+\frac{z f_{0}^{\prime \prime}(z)}{f_{0}^{\prime}(z)} \neq i b \quad(z \in E),
$$

where $b$ is real and $|b| \geq 3^{1 / 2} p$.

On the other hand, it is well-known that if $f(z) \in S(p)$, then for $|z|=$ $r<1$,

$$
\frac{p(1-r) r^{p-1}}{(1+r)^{2 p+1}} \leq\left|f^{\prime}(z)\right| \leq \frac{p(1+r) r^{p-1}}{(1-r)^{2 p+1}} .
$$

Since

$$
\left|f_{0}^{\prime}(r)\right|>\frac{p(1+r) r^{p-1}}{(1-r)^{2 p+1}} \quad(p \geq 2,0<r<1)
$$

it follows that $f_{0}(z) \notin S(p)$.

In this paper, we shall correct and extend the main theorem of [3]. 


\section{Results}

We need the following lemma due to Miller and Mocanu [1].

Lemma. Let $g(z)$ be analytic and univalent in $E$ and let $\theta(w)$ and $\varphi(w)$ be analytic in a domain $D$ containing $g(E)$, with $\varphi(w) \neq 0$ when $w \in g(E)$. Set

$$
Q(z)=z g^{\prime}(z) \varphi(g(z)), \quad h(z)=\theta(g(z))+Q(z)
$$

and suppose that

(i) $Q(z)$ is univalent and starlike in $E$, and

(ii) $\operatorname{Re} \frac{z h^{\prime}(z)}{Q(z)}=\operatorname{Re}\left\{\frac{\theta^{\prime}(g(z))}{\varphi(g(z))}+\frac{z Q^{\prime}(z)}{Q(z)}\right\}>0 \quad(z \in E)$.

If $P(z)$ is analytic in $E$, with $P(0)=g(0), P(E) \subseteq D$ and

$$
\theta(P(z))+z P^{\prime}(z) \varphi(P(z)) \prec \theta(g(z))+z g^{\prime}(z) \varphi(g(z))=h(z)
$$

then

$$
P(z) \prec g(z),
$$

where the symbol $\prec$ denotes subordination, and $g(z)$ is the best dominant of $(3)$.

Applying the above lemma, we derive

Theorem 1. If $f(z) \in A(p)$ satisfies

$$
(1-\alpha) \frac{z f^{\prime}(z)}{f(z)}+\alpha\left(1+\frac{z f^{\prime \prime}(z)}{f^{\prime}(z)}\right) \neq i b \quad(z \in E),
$$

where $\alpha>0, b$ is a real number and

$$
|b| \geq(\alpha(\alpha+2 p))^{1 / 2}
$$

then $f(z) \in S(p)$.

Proof. Let us put

$$
P(z)=\frac{z f^{\prime}(z)}{f(z)}
$$

where $P(0)=p$. From (4) and using the same argument as [3, p. 133], we easily have $P(z) \neq 0$ in $E$. 
From (6) we obtain

$$
(1-\alpha) \frac{z f^{\prime}(z)}{f(z)}+\alpha\left(1+\frac{z f^{\prime \prime}(z)}{f^{\prime}(z)}\right)=P(z)+\alpha \frac{z P^{\prime}(z)}{P(z)} .
$$

Using (4), (5) and (7), we deduce that

$$
P(z)+\alpha \frac{z P^{\prime}(z)}{P(z)} \prec p\left(\frac{1+z}{1-z}+\frac{\alpha}{p} \frac{2 z}{1-z^{2}}\right) .
$$

Set

$$
g(z)=p \frac{1+z}{1-z}, \quad \theta(w)=w, \quad \varphi(w)=\frac{\alpha}{w}
$$

and $D=\{w: w \neq 0\}$ in the lemma. Then the function

$$
Q(z)=\frac{\alpha z g^{\prime}(z)}{g(z)}=\frac{2 \alpha z}{1-z^{2}}
$$

is univalent and starlike in $E$. Also,

$$
h(z)=g(z)+Q(z)=p \frac{1+z}{1-z}+\frac{2 \alpha z}{1-z^{2}}
$$

and

$$
\operatorname{Re} \frac{z h^{\prime}(z)}{Q(z)}=\operatorname{Re}\left\{\frac{p}{\alpha} \frac{1+z}{1-z}+\frac{1+z^{2}}{1-z^{2}}\right\}>0 \quad(z \in E) .
$$

In view of (8), the lemma yields

$$
P(z) \prec p \frac{1+z}{1-z} .
$$

This shows that $f(z) \in S(p)$ and the proof is complete.

From Theorem 1, we easily have the following results.

Corollary 1. If $\alpha>0$ and $f(z) \in A(p)$ satisfies

$$
\left|\operatorname{Im}\left\{(1-\alpha) \frac{z f^{\prime}(z)}{f(z)}+\alpha \frac{z f^{\prime \prime}(z)}{f^{\prime}(z)}\right\}\right|<(\alpha(\alpha+2 p))^{1 / 2} \quad(z \in E),
$$

then $f(z) \in S(p)$.

Corollary 2. If $\alpha>0$ and $f(z) \in A(p)$ satisfies

$$
\left|(1-\alpha) \frac{z f^{\prime}(z)}{f(z)}+\alpha\left(1+\frac{z f^{\prime \prime}(z)}{f^{\prime}(z)}\right)-p\right|<p+\alpha \quad(z \in E),
$$


then $f(z) \in S(p)$.

For $\alpha=1$, Corollary 2 leads to the assertion: If $f(z) \in A(p)$ satisfies $\left|1+\frac{z f^{\prime \prime}(z)}{f^{\prime}(z)}-p\right|<p+1 \quad(z \in E)$, then $f(z) \in S(p)$.

Corollary 3. If $f(z) \in A(p)$ satisfies

$$
1+\frac{z f^{\prime \prime}(z)}{f^{\prime}(z)} \neq i b \quad(z \in E)
$$

where $b$ is real and

$$
|b| \geq(1+2 p)^{1 / 2}
$$

then $f(z) \in S(p)$.

Next, we derive

Theorem 2. If $\alpha>0$ and $f(z) \in A(p)$ satisfies

$$
\left|(1-\alpha) \frac{z f^{\prime}(z)}{f(z)}+\alpha\left(1+\frac{z f^{\prime \prime}(z)}{f^{\prime}(z)}\right)-p\right|<p+\frac{\alpha}{2} \quad(z \in E),
$$

then

$$
\left|\frac{z f^{\prime}(z)}{f(z)}-p\right|<p \quad(z \in E)
$$

Proof. According to Corollary 2 the function $f(z)$ belongs to $S(p)$.

Let

$$
P(z)=\frac{z f^{\prime}(z)}{f(z)} .
$$

Then by (7), the assumption (9) becomes

$$
\left|P(z)+\alpha \frac{z P^{\prime}(z)}{P(z)}-p\right|<p+\frac{\alpha}{2} \quad(z \in E) .
$$

Set

$$
g(z)=p(1+z), \quad \theta(w)=w, \quad \varphi(w)=\alpha / w
$$

and $D=\{w: w \neq 0\}$ in the lemma. Then we have

$$
Q(z)=\frac{\alpha z g^{\prime}(z)}{g(z)}=\frac{\alpha z}{1+z}
$$




$$
h(z)=g(z)+Q(z)=p(1+z)+\frac{\alpha z}{1+z}
$$

and

$$
\operatorname{Re} \frac{z h^{\prime}(z)}{Q(z)}=\operatorname{Re}\left\{\frac{p}{\alpha}(1+z)+\frac{1}{1+z}\right\}>\frac{1}{2}(z \in E) .
$$

For $|z|=1$ and $z \neq-1$,

$$
|h(z)-p| \geq p+\alpha \operatorname{Re} \frac{1}{1+z}=p+\frac{\alpha}{2} .
$$

Thus it follows from (11) that

$$
P(z)+\alpha \frac{z P^{\prime}(z)}{P(z)} \prec h(z) .
$$

The lemma now leads to $P(z) \prec p(1+z)$, which gives (10).

When $p=1$, Mocanu [2] proved Theorem 2 by a different method.

\section{REFERENCES}

1. S. S. Miller and P. T. Mocanu, On some classes of first-order differential subordinations, Michigan Math. J. 32 (1985), 185-195.

2. P. T. Mocanu, Some integral operators and starlike functions, Rev. Roumaine Math. Pures Appl. 31 (1986), 231-235.

3. M. Nunokawa, On a sufficient condition for multivalently starlikeness, Tsukuba J. Math. 18 (1994), 131-134.

Department of Mathematics, Suzhou University Suzhou, Jiangsu 215006, China 